\title{
Isolating the Role of the Node-Linker Bond in the Compression of UiO-66 Metal-Organic Frameworks
}

\author{
Louis R. Redfern, ${ }^{1}$ Maxime Ducamp, ${ }^{2}$ Megan C. Wasson, ${ }^{1}$ Lee Robison, ${ }^{1}$ Florencia A. Son, ${ }^{1}$ François- \\ Xavier Coudert, ${ }^{2 *}$ and Omar K. Farha ${ }^{*}$
}

1. International Institute of Nanotechnology and Department of Chemistry, Northwestern University, 2145 Sheridan Road, Evanston, Illinois 60208, United States.

2. Chimie ParisTech, PSL University, CNRS, Institut de Recherche de Chimie Paris, 75005, Paris, France.

\begin{abstract}
Understanding the mechanical properties of metal-organic frameworks (MOFs) is essential to the fundamental advancement and practical implementations of porous materials. Recent computational and experimental efforts have revealed correlations between mechanical properties and pore size, topology, and defect density. These results demonstrate the important role of the organic linker in the response of these materials to physical stresses. However, the impact of the coordination bond between the inorganic node and organic linker on the mechanical stability of MOFs has not been thoroughly studied. Here, we isolate the role of this node-linker coordination bond to systematically study the effect it plays in the compression of a series of isostructural MOFs, M-UiO-66 (M = Zr, Hf, or Ce). The bulk modulus (i.e. the resistance to compression under hydrostatic pressure) of each MOF is determined by in situ diamond anvil cell (DAC) powder X-ray diffraction measurements and density functional theory (DFT) simulations. These experiments reveal distinctive behavior of Ce-UiO-66 in response to pressures under one GPa. In situ DAC Raman spectroscopy and DFT calculations support the observed differences in compressibility between $\mathrm{Zr}$-UiO-66 and the Ce- analogue. Monitoring changes in bond lengths as a function of pressure through DFT simulations provides a clear picture of those which shorten more drastically under pressure and those which resist compression. We hypothesize that the presence of $\sim 10 \% \mathrm{Ce}^{3+}$ in the nodes of Ce-UiO-66 may contribute to the weakening of the node-linker coordination, manifesting in the distinct behavior under pressure. This study demonstrates that changes to the node-linker bond can have significant ramifications on the mechanical properties of MOFs.
\end{abstract}

\section{INTRODUCTION}

Over the past two decades, metal-organic frameworks (MOFs) have become a prominent class of porous materials for many applications, including gas adsorption, ${ }^{1-2}$ chemical separations, ${ }^{3-4}$ and heterogeneous catalysis. ${ }^{5-7}$ These scaffolds consist of inorganic nodes connected by multitopic organic linkers to form 2- and 3-dimensional frameworks with exceptional porosity and tremendous structural diversity. ${ }^{8}$ While several classes of MOFs have demonstrated high chemical and thermal stability in recent years, ${ }^{9}$ a rigorous understanding of how these materials respond to mechanical stress is still emerging. Interesting phenomena, such as pressure-induced amorphization, ${ }^{10}$ pore collapse, ${ }^{11}$ and structural transitions, ${ }^{12}$ have been well documented in MOFs when exposed to pressures on the order of $1 \mathrm{GPa}$. Elucidating structure-property relationships of MOFs based upon their mechanical properties is challenging due to the sheer number of frameworks and the highly specialized equipment required for experimental measurements. On the other hand, high-throughput computational studies offer insight into the structural characteristics that relate to mechanical properties, ${ }^{13}$ though detailed design principles remain elusive. A combination of experimental and theoretical work provides a more nuanced picture of the factors that dictate the mechanical properties of MOFs.
UiO-66 is among the most well-studied MOFs due to its cubic space group, remarkable chemical and thermal stability. ${ }^{14}$ The structure consists of 12 -connected $\operatorname{Zr}_{6}\left(\mu_{3}-0\right)_{4}\left(\mu_{3}-\right.$ $\mathrm{OH})_{4}$ nodes joined by linear 1,4-benzenedicarboxylic acid linkers which assemble into the fcu topology (Figure 1). ${ }^{15}$ Several studies have demonstrated that UiO-66 is more resistant to mechanical stress than other prototypical MOFs, ${ }^{16-17}$ though the presence of missing linker defects can significantly impact the mechanical properties. ${ }^{18-21}$ In a recent study from our group, we demonstrated that systematic extension of the organic linker in the UiO family of MOFs leads to a decrease in the bulk modulus (that is, the resistance to hydrostatic pressure; $\left.K=-V(\partial P / \partial V)_{T}\right){ }^{22}$ Given the breadth of knowledge regarding this framework, UiO-66 is a well-established platform for investigating the mechanism of structural changes at high pressures. While numerous reports support the notion that changes to the organic linker ${ }^{23-25}$ and topology ${ }^{26-27}$ can impact the compressibility of MOFs, systematic studies into the effect of changes to the metal node are less prevalent. ${ }^{28-30} \mathrm{UiO}-66$ provides an excellent platform to investigate these trends, as it is a robust 


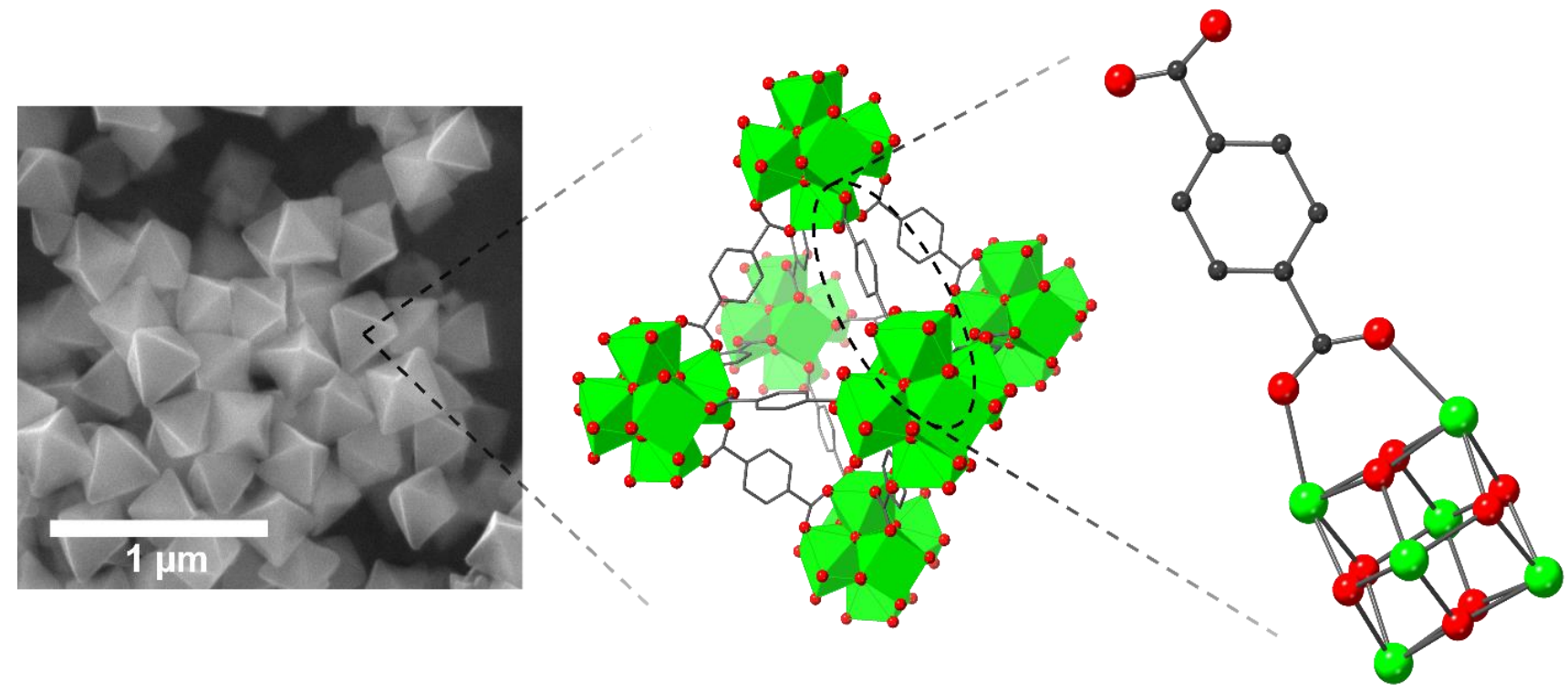

Figure 1. The octahedral cages of UiO-66 are comprised of $\mathrm{M}_{6}(\mathrm{M}=\mathrm{Zr}$, Hf, or Ce) nodes connected by 1,4-benzenedicarboxylic acid linkers. Hydrogen atoms are omitted for clarity.

framework that is known to retain its crystallinity at pressures greater than $1 \mathrm{GPa}^{28}$

Herein, we investigate the role of the metal-carboxylate bond in the compression of UiO-66 by varying the identity of the metal node from $\mathrm{Zr}$-UiO-66 to Hf-UiO-66 and Ce-UiO66. By keeping the topology, linker, and experimental conditions constant, we isolate the coordination bond as the single structural variable that changes in this series of MOFs. While the $\mathrm{Zr}$ and $\mathrm{Hf}$ analogues exhibit almost identical behavior under pressure, Ce-UiO-66 is found to be much more compressible. Pressure-dependent Raman spectroscopy supports the observations made from high-pressure in situ powder X-ray diffraction (PXRD) measurements. Density Functional Theory (DFT) simulations of Zr- and Ce-UiO66 are in good agreement with the experimental observations and reveal that the node-linker bond of Ce-UiO-66 compresses more readily than that of the $\mathrm{Zr}$ analogue. Together, these results indicate that the identity of the metal node in MOFs can have a significant impact on their response to high pressures by modulating the strength of the coordination bonds that hold these scaffolds together.

\section{EXPERIMENTAL METHODS}

All MOFs were synthesized and activated according to modified literature procedures to minimize the presence of defects. ${ }^{31-33}$ The materials were characterized using ambient pressure powder X-ray diffraction (PXRD) and isothermal nitrogen adsorption to verify their crystallinity and porosity, respectively (Figures S1 and S2). X-ray photoelectron spectroscopy (XPS) was conducted with an $\mathrm{Al} \mathrm{K}_{\alpha}$ source. Five scans lasting 30 seconds each were collected. Ambient pressure Raman spectroscopy was conducted using a $532 \mathrm{~nm}$ excitation laser (see Supporting Information for details). High-pressure PXRD was conducted at the 17BM-B beamline $(\lambda=0.45390 \AA)$ at the Advanced Photon Source (APS) at Argonne National Laboratory (ANL) using a diamond anvil cell (DAC) sample environment. In a typical experiment, the MOF sample was gently ground with an internal standard, $\mathrm{CaF}_{2}$, to ensure uniform mixing and to break up any large crystals. The mixture was loaded into a hole in a pre-indented stainless-steel gasket and sealed in the DAC. An ambient pressure diffraction pattern was collected, then a drop of Fluorinert ${ }^{\mathrm{TM}} \mathrm{FC}-70$ was added to the cell as a non-penetrating pressure transmitting fluid. In situ PXRD data were then collected as a function of pressure. Unit cell parameters were then extracted from the PXRD patterns using Le Bail refinement. Bulk moduli $\left(\mathrm{K}_{0}=-\mathrm{V}\right.$ $\partial \mathrm{P} / \partial \mathrm{V}$ ) were extracted by fitting a plot of unit cell volumes vs. pressure to a second-order Birch-Murnaghan equation of state. Pressure-dependent Raman spectroscopy experiments were conducted at the GSECARS offline Raman spectrometer located at the APS at ANL. ${ }^{34}$ Spectra were collected using a $532 \mathrm{~nm}$ excitation laser, and ruby fluorescence was used to monitor pressure inside the DAC. For further details regarding DAC diffraction and Raman experiments, see Supporting Information.

Each MOF was investigated through computational simulations using the Density Functional Theory (DFT) as implemented in the CRYSTAL14 code. ${ }^{35}$ All-electron localized basis sets were used for all the atoms except for hafnium and cerium, for which a pseudopotential approach was used. These basis sets can be found on the CRYSTAL online library with the corresponding acronyms and original references: C: C_G-31d1G_gatti_1994;36 0: basis set used by Vlenzano et al.;15 H: H_3-1p1G_1994;36 Zr: Zr_all_electron_dovesi_unpub; ${ }^{15}$ Hf: Hf_ECP_stevens_411d31G_munoz_2007; ${ }^{37}$ Ce: Ce_ECP_Meyer_2009.38

Several different functionals, at different levels of approximation, were considered to describe the exchange and correlation components of the energy. Among these, PBESOL0 hybrid functional ${ }^{39}$ was retained as it gives a good accuracy while keeping an acceptable computational cost. The effect of Grimme-type dispersion corrections ${ }^{40}$ was tested and found to be of a minor impact on the optimized structures and derived properties. Therefore, these corrections were not included in this study. For further computational details, see Supporting Information.

Mesh sampling of the reciprocal space was performed using the Monkhorst-Pack scheme. ${ }^{41}$ Given the size of the 
systems, a $k$-point mesh of $2 \times 2 \times 2$ was used for each MOF structure to obtain convergence of the properties of interest. This generated mesh was then used for all our calculations. Stricter convergence criteria than the defaults proposed by the CRYSTAL14 code were used for geometry optimization (a maximum of 0.0005 a.u. on atomic displacements during one optimization step and 0.0001 a.u. on forces). Optimized structures and representative input files for the calculations are available online at https://github.com/fxcoudert/citable-data

\section{RESULTS AND DISCUSSION}

Ambient pressure PXRD patterns for each MOF are in good agreement with the simulated powder patterns (Figure S1) from reported crystal structures, ${ }^{32-33}$ indicating that the Ui0-66 structure is retained when synthesized with different metals. Nitrogen physisorption isotherms (Figure S2) indicate that porosity of the UiO-66 MOFs is also maintained, though evidence of a small number of missing linker defects (estimated to be $6-13 \%$ ) is present in the isotherm of Ce-UiO-66. A detailed discussion of the defect density in Ce-UiO-66 can be found in the supporting information.

Upon applying pressure using a DAC, the PXRD peaks shift steadily to higher angles of diffraction, indicating a decrease in unit cell volume (Figures S4 and S5, Tables S1 and S2). We extracted the unit cell volumes of each MOF at each measured pressure. Plotting the unit cell volumes of Zr-UiO-66, Hf-UiO-66, and Ce-UiO-66 as a function of pressure reveals that the $\mathrm{Zr}$ and $\mathrm{Hf}$ analogues behave nearly identically over a pressure range of $0-0.4 \mathrm{GPa}$, followed by a slight divergence above $0.4 \mathrm{GPa}$ (Figure 2). Remarkably, the unit cell volume of Ce-UiO-66 decreases much more rapidly than the other MOFs, with noticeable discontinuities around $0.1 \mathrm{GPa}$ and $0.4 \mathrm{GPa}$. These discontinuities may indicate partial amorphization or possible pressure-induced phase transitions, i.e. changes to the MOF structure upon achieving certain pressures that alter the mechanical properties of the material. Unfortunately, the PXRD patterns collected were of insufficient quality to determine the nature of these suspected structural transformations.

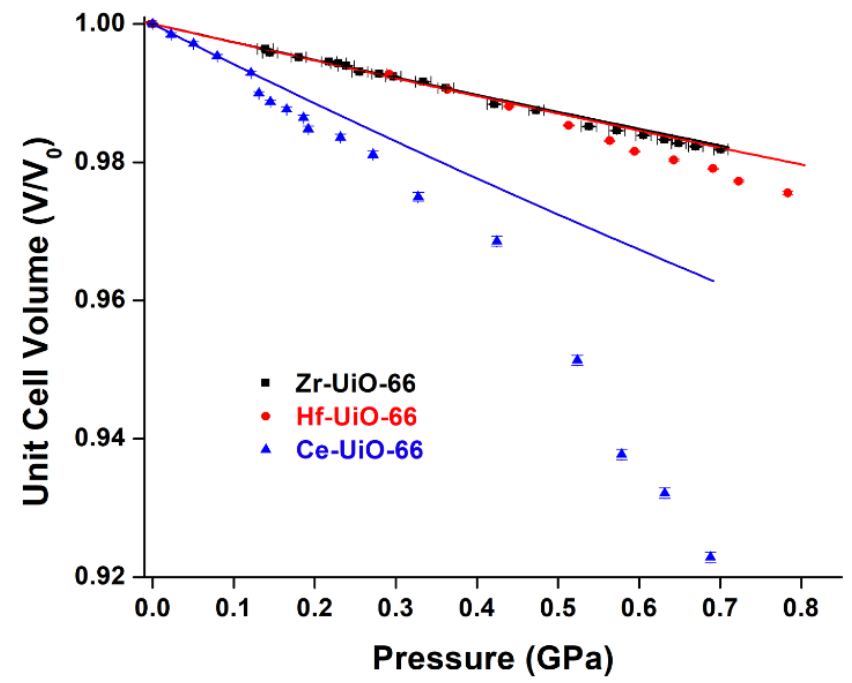

Figure 2. Unit cell compression of M-UiO-66. Solid curves represent the second-order Birch Murnaghan equation of state used to determine the bulk modulus of each MOF. Data for $\mathrm{Zr}$ UiO-66 are reproduced with permission from Ref. 22
The initial data points for each MOF were fit to a secondorder Birch-Murnaghan equation of state to estimate the bulk modulus $\left(K=-V(\partial P / \partial V)_{T}\right)$ of the material (Figure 2). The bulk modulus describes the pressure required to induce a given change in volume and is inversely related to compressibility. Zr-UiO-66 and Hf-UiO-66 exhibit nearly identical bulk moduli $\left(K_{0}=37.9 \pm 0.6 \mathrm{GPa}^{22}\right.$ and $37 \pm 1 \mathrm{GPa}$, respectively), while Ce-UiO-66 has a much lower bulk modulus $\left(K_{0}=16.9 \pm 0.7 \mathrm{GPa}\right)$ as evidenced by the steep decline in unit cell volume with pressure. These results indicate that while $\mathrm{Zr}-\mathrm{UiO}-66$ and Hf-UiO-66 resist compression to the same degree, the Ce framework is significantly less rigid. Because the three MOFs have similar properties at ambient conditions and share identical organic linker and topology, we attribute the distinct mechanical properties to differences in the bond between the node and linker.

Given the striking difference in the bulk modulus of CeUiO-66, we turned to pressure-dependent Raman spectroscopy to probe the coordination bond between the metal node and organic linker. Unfortunately, the node-linker bond cannot be directly monitored using this technique because this vibration is infrared active. To circumvent this challenge, we selected the $\mathrm{C}$-C stretch between the aromatic core of the linker and the carboxylate carbon and the $\mathrm{O}-\mathrm{C}-\mathrm{O}$ symmetric stretch of the carboxylate (Figure S6) as target vibrations which provide insight into the strength of the metal-linker bond and have been assigned previously. ${ }^{42}$

Ambient pressure Raman spectra were collected for each material to determine the frequencies that correspond to the vibrations of interest. Then, Raman spectra were collected within a DAC with incremental increases in pressure for each MOF (Figure 3). In each sample, significant peak broadening is observed with increasing pressure, which is a common phenomenon in high-pressure Raman spectroscopy.43 In Zr-UiO-66, both vibrations of interest exhibit a steady hypsochromic shift as pressure increases from 0 1.20 GPa. Hf-UiO-66 displays a similar behavior over a pressure range of $0-0.92 \mathrm{GPa}$, though the effect in the $\mathrm{C}-\mathrm{C}$ stretch (starting at $1437.8 \pm 0.7 \mathrm{~cm}^{-1}$ ) is somewhat obscured by peak broadening. The Raman spectrum of Ce-UiO-66 changes dramatically between ambient pressure and the spectrum collected under pressure $(0.24 \mathrm{GPa})$. Immediate broadening of the two peaks results in a single broad signal, precluding meaningful qualitative analysis. This significant change in the shape of the Raman spectrum may result from structural changes occurring at low pressures. The stark difference in behavior between Ce-UiO-66 and the $\mathrm{Zr}$ and $\mathrm{Hf}$ counterparts supports the observed contrast in compression from the PXRD data.

We then fit the region of the Raman spectra containing the vibrations of interest with two pseudo-Voigt functions in order to analyze the observed changes in a more quantitative manner. The frequencies corresponding to the center of each peak are shown in Figure 4 as a function of increasing pressure. In all three MOFs, the C-C stretch shifts steadily to higher frequencies in general, with a slight discontinuity present around 0.4 GPa in Ce-UiO-66 (Figure 4a). This small jump in the Raman spectrum coincides with the dramatic change in compressibility observed in the DAC PXRD data. We attribute these discontinuities to possible structural changes or partial amorphization that occur around 

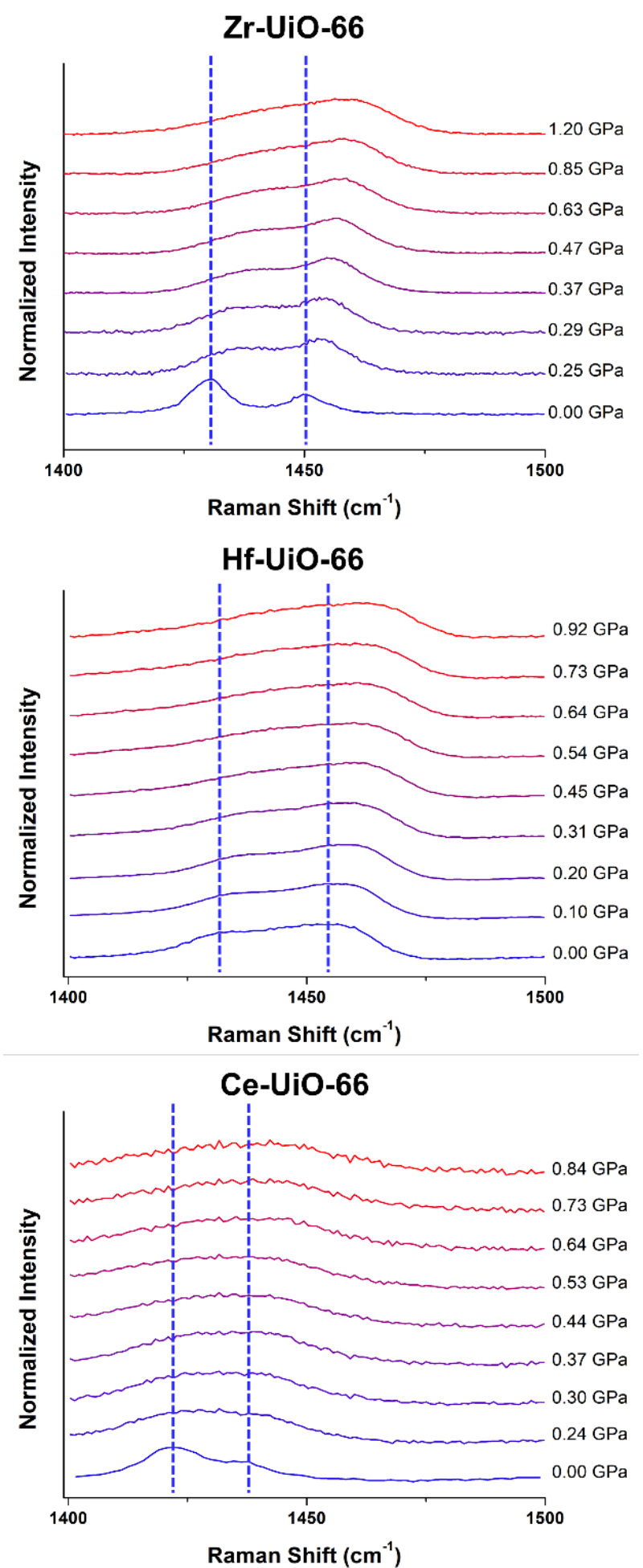

Figure 3. Raman spectra of Zr-UiO-66, Hf-UiO-66, and CeUiO-66 as a function of pressure. Dashed vertical lines are centered on the ambient pressure peak maxima and are included as a guide to the eye.

$0.4 \mathrm{GPa}$, though further characterization is required to elucidate the details of such a transformation. For Zr-UiO-66 and Hf-UiO-66, the O-C-O symmetric stretch steadily increases in frequency with increasing pressure; however, in Ce-UiO-66 this vibration remains almost invariant after an initial increase in frequency upon pressurizing to $0.24 \mathrm{GPa}$. The consistency of this Raman shift indicates that the bond
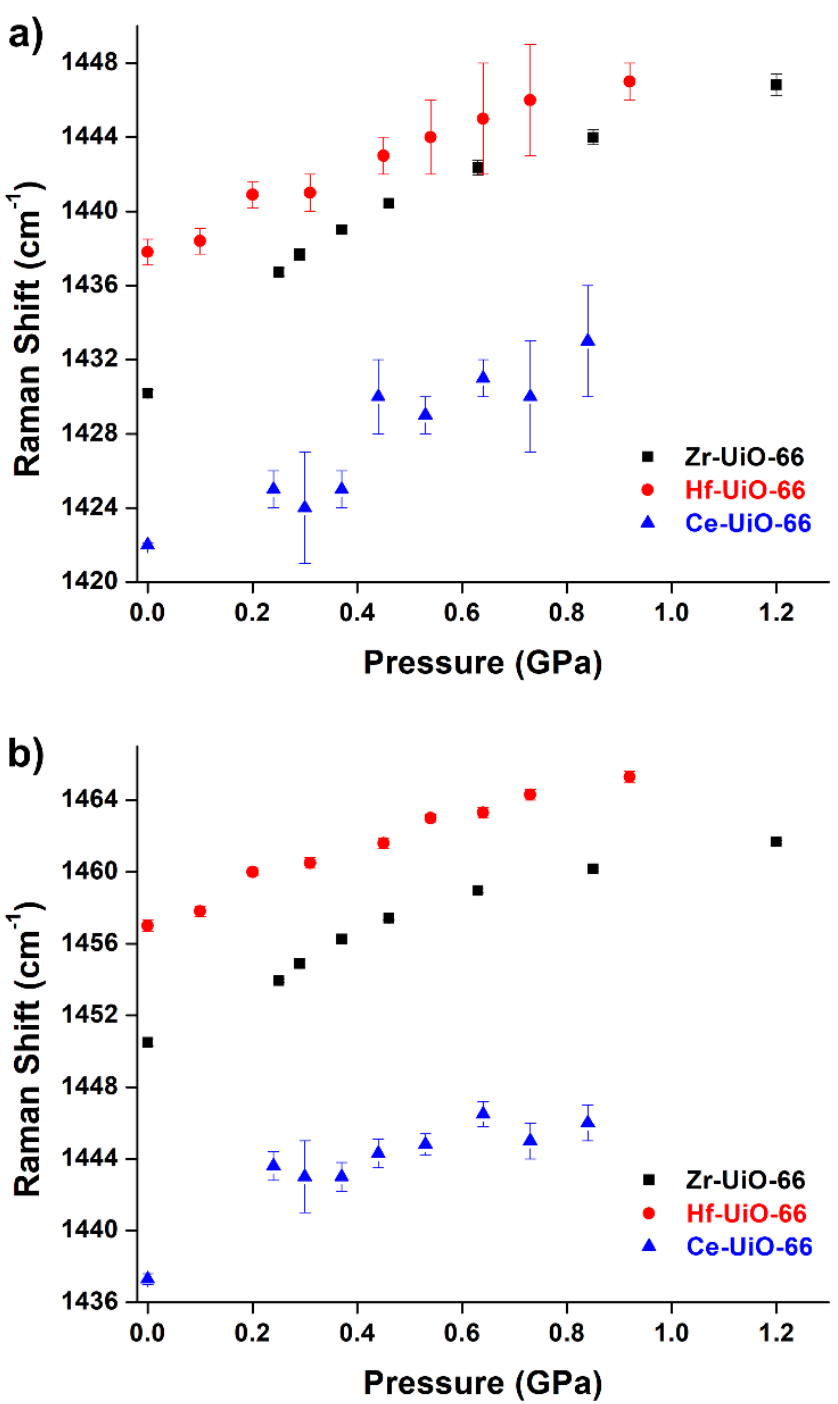

Figure 4. Frequencies of the (a) C-C stretch between carboxylate group and aromatic core of the UiO-66 linker and (b) 0$\mathrm{C}-\mathrm{O}$ symmetric stretch of the carboxylate group as a function of pressure.

lengths and vibrational frequency of the carboxylate group in Ce-UiO-66 do not change as rapidly as the $\mathrm{Zr}$ and $\mathrm{Hf}$ analogues. The distinct behavior of the $\mathrm{O}-\mathrm{C}-\mathrm{O}$ vibration in $\mathrm{Ce}-$ UiO-66 is evidence of the role of the metal-carboxylate bond in the pressure response of the material. Suslick and coworkers observed breakage of the metal-carboxylate bond in $\mathrm{Zr}-\mathrm{UiO}-66$ at high pressures by EXAFS, and we propose that a similar mechanism could be operative here. ${ }^{44}$

In order to better understand the compression of $\mathrm{Zr}$-UiO66 and Ce-UiO-66, we performed DFT calculations of their structures, their elastic properties, and their vibrational modes in the harmonic approximation. First, the experimental structures were optimized with full use of symmetry, relaxing both atomic positions and unit cell parameters. Very good agreement was found with the experimental cell parameters (Table S6). We then performed frequency calculations in the harmonic approximation in order to determine the characteristics of the MOFs' vibration modes, in particular the $\mathrm{C}-\mathrm{C}$ stretch and $\mathrm{O}-\mathrm{C}-\mathrm{O}$ symmetric stretch. 
Again, a good agreement with the experimental results was observed for both compounds (Table S7).

We note here a few negative frequencies are observed in the case of Ce-UiO-66; those vibration modes are related to the ligand bowing out of its average plane, also called "guitar string" modes. These vibrational modes have been observed in UiO-compounds, as well as in other MOFs, ${ }^{24}$ and arise from the fact that DFT calculations are performed at 0 $\mathrm{K}$ on a crystallographic structure with high symmetry, which is an average structure. At finite temperature, the ligand would bow out of the plane in two directions, in this low-frequency mode, giving an in-plane average position which is the high-symmetry structure observed by X-ray diffraction.

Determination of the stiffness tensor (i.e., the second-order elastic constants) through linear response calculations allowed us to derive the bulk modulus for each material. Reproducing the experimental trend, simulations confirm a different compression between $\mathrm{Zr}-\mathrm{UiO}-66$ and its Ce analogue, with values of 42 and $37 \mathrm{GPa}$ respectively. Although the difference is not as drastic as is observed in the experimental measurements, it demonstrates the softer nature of the Ce-UiO-66 framework.

We then conducted calculations of the structural evolution of the frameworks under pressure: geometry optimizations were performed in the pressure range of 0 to $2 \mathrm{GPa}$. Summary of the cell parameters for each pressure can be found in the supporting information (Table S6), as well as the optimized structures. As found experimentally, we observed a linear variation of the volume with respect to the pressure for Zr-UiO-66. However, our DFT calculations cannot reproduce the possible phase transition experimentally observed at 0.4 GPa for Ce-UiO-66 - indicating that it involves a symmetry-breaking transition to a lower-symmetry phase. In the case of Ce-UiO-66, computational results show a linear variation like that of the $\mathrm{Zr}$ analogue, though the slope is steeper, confirming the lower bulk modulus measured and calculated for this MOF.

From the stressed structures, we highlight a few structural variations of chemical importance. Considering first the ligand, a few similarities are observed between $\mathrm{Zr}$ - and Ce-UiO-66: variation of the $\mathrm{O}-\mathrm{C}-\mathrm{O}$ angle as well as the dihedral angle between ligands was identical in both materials, shifting around $1^{\circ}$ in each case. However, using the cell parameter as a reference by plotting $\left(l / l_{0}\right) /\left(a / a_{0}\right)(l=$ bond length, $l_{0}=$ bond length at ambient pressure, $a=$ unit cell parameter, $a_{0}=$ unit cell parameter at ambient pressure) (Figure 5), we found that $\mathrm{C}-\mathrm{O}$ bond decreases more slowly than the lattice parameter in both cases $\left[\left(l / l_{0}\right) /\left(a / a_{0}\right)>1\right.$ as pressure increases] whereas $\mathrm{C}-\mathrm{C}$ bond decreases almost exactly at the same rate $\left[\left(l / l_{0}\right) /\left(a / a_{0}\right) \approx 1\right.$ as pressure increases]. Moreover, we saw that this $\mathrm{C}-\mathrm{O}$ bond shortens faster in the case of $\mathrm{Zr}$ supporting the slower variation of the $\mathrm{O}-\mathrm{C}-\mathrm{O}$ symmetric stretch for Ce. This demonstrates the importance of the carboxylate group to understand the compression of this material. Metal-carboxylate distances were also investigated in both materials and we found that they show a faster decrease than the lattice parameter $\left[\left(l / l_{0}\right) /\left(a / a_{0}\right)<1\right.$ as pressure increases]. We also saw that this decrease is even more pronounced in the case of $\mathrm{Ce}$ UiO-66 which could be due to the orbitals of Ce being more

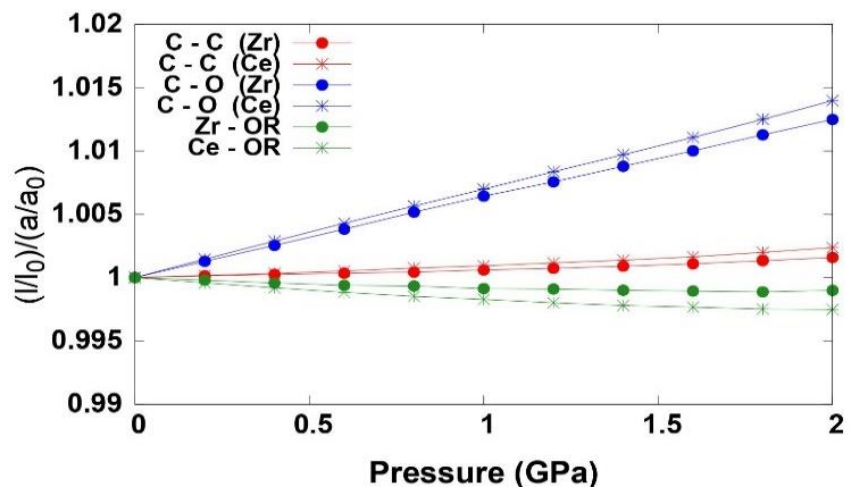

Figure 5. $\left(\mathrm{l} / \mathrm{l}_{0}\right) /\left(\mathrm{a} / \mathrm{a}_{0}\right)$ as a function of pressure for bond lengths involving the linker carboxylate: C-C bond (red), C-O bond (blue), and M-OR bond (green, $\mathrm{M}=\mathrm{Zr}$ or $\mathrm{Ce}, \mathrm{R}=$ linker). Filled circles represent bond lengths of $\mathrm{Zr}-\mathrm{UiO}-66$ and stars represent bond lengths of $\mathrm{Ce}-\mathrm{UiO}-66$.

diffuse than those of $\mathrm{Zr}$, leading then to a slightly higher flexibility of the Ce-carboxylate bond. While these simulations were conducted in the zero temperature limit, we expect the observed trends to be valid at room temperature given the relatively modest thermal expansion exhibited by $\mathrm{UiO}$ 66.45

Given the apparent role of the metal-carboxylate bond in determining the compressibility of these MOFs, we considered the possibility of $\mathrm{Ce}^{3+}$ forming during the MOF synthesis due to the high reduction potential of $\mathrm{Ce}^{4+}$. This $\mathrm{Ce}^{3+}$ species could then incorporate into the Ce-UiO-66 structure accompanied by an additional proton (to maintain charge balance) on one of the $\mu_{3}-\mathrm{O}$ groups present in the $\mathrm{Ce}_{6}$ node. To quantify the portion of reduced $\mathrm{Ce}$, we conducted XPS experiments, revealing $\sim 10 \% \mathrm{Ce}^{3+}$ and $\sim 90 \% \mathrm{Ce}^{4+}$ present in Ce-UiO-66 (Figure S7). This ratio corresponds to roughly $47 \%$ of $\mathrm{Ce}_{6}$ nodes containing at least one $\mathrm{Ce}^{3+}$ atom, assuming they are evenly distributed throughout the MOF. Even with a low proportion of $\mathrm{Ce}^{3+}$, a significant number of nodes likely contain a reduced ion. $\mathrm{Ce}^{3+}$ exhibits a longer ionic radius than $\mathrm{Ce}^{4+}$, which is often invoked to rationalize the well-documented unit cell expansion of bulk $\mathrm{CeO}_{2}$ upon reduction. ${ }^{46-47}$ The presence of $\mathrm{Ce}^{3+}$ in the nodes of Ce-UiO-66 may lead to distortions of the node structure of Ce-UiO-66, influencing the mechanical properties of the MOF, and may also contribute to the lowering of the elastic moduli of the Ce-UiO-66 due to the softer Ce-carboxylate coordination allowing easier compression and shearing deformations. These rationales are in agreement with the difference observed between experimental and computational results on the bulk modulus of Ce-UiO-66, as calculations were performed on an ideal system with $100 \% \mathrm{Ce}^{4+}$. We hypothesize that the prevalence of $\mathrm{Ce}^{3+}$ throughout Ce-UiO-66 contributes to the uniquely distinct behavior of the framework under pressure compared to the $\mathrm{Zr}$ - and Hf- analogues.

\section{CONCLUSIONS}

This study investigates the influence of metal identity on the compression of a prototypical MOF, UiO-66. We conducted in situ DAC PXRD experiments to quantify the bulk modulus for three materials: $\mathrm{Zr}-\mathrm{UiO}-66$, Hf-UiO-66, and CeUiO-66. These results indicate that Ce-UiO-66 compresses much more readily than the $\mathrm{Zr}$ and $\mathrm{Hf}$ analogues (Figure 2). We then conducted in situ DAC Raman spectroscopy to 
probe two vibrations involving the carboxylate group of the organic linker. While the Raman shifts of the C-C stretch between the carboxylate and aromatic core of the linker increase in frequency with pressure for all three MOFs, the 0C-O symmetric stretch of Ce-UiO-66 remains nearly constant from 0.24-0.84 GPa. This behavior contrasts the steady hypsochromic shift observed for the same vibration in Zr-UiO-66 and Hf-UiO-66 (Figure 4). DFT simulations reveal the important role of the inorganic node in influencing the compression of individual bond lengths upon exposure to high pressures. We hypothesize that the presence of $\sim 10 \% \mathrm{Ce}^{3+}$ in the nodes of Ce-UiO-66 may significantly impact the behavior of this MOF under pressure.

Changing the metal that comprises the nodes of a MOF has been shown previously to influence the electronic properties, ${ }^{48}$ catalytic activity, ${ }^{49}$ and chemical stability ${ }^{50}$ of the material. Here, we have presented an interesting example in which altering the metal node of a MOF can have significant impact on the mechanical properties of the framework. While the response of $\mathrm{Zr}-\mathrm{UiO}-66$ and Hf-UiO-66 to pressure is nearly identical, the Ce analogue exhibits drastically different behavior despite the isostructural nature of the three MOFs. These results indicate that the coordination bond between the linker and node of UiO-66 plays a role in the compression of the materials. This study provides insight into how these porous scaffolds respond to high pressures and the structural properties that can be adjusted to modulate the mechanical response of MOFs.

\section{ASSOCIATED CONTENT}

Supporting Information. Materials synthesis, ambient condition characterization, diamond anvil cell experimental details, and computational details. "This material is available free of charge via the Internet at http://pubs.acs.org."

\section{AUTHOR INFORMATION}

\section{Corresponding Authors}

* François-Xavier Coudert - Email: fx.coudert@chimieparistech.psl.eu

* Omar K. Farha - Email: o-farha@northwestern.edu

\section{Author Contributions}

The manuscript was written through contributions of all authors.

\section{ACKNOWLEDGMENT}

O.K.F. gratefully acknowledges support from the Defense Threat Reduction Agency (HDTRA1-19-1-0007). M.D. and F.X.C. acknowledge financial support from the Agence Nationale de la Recherche under project "MATAREB" (ANR-18-CE290009-01) and access to high-performance computing platforms provided by GENCI grant A0070807069. Portions of this work were performed at GeoSoilEnviroCARS (The University of Chicago, Sector 13), Advanced Photon Source (APS), Argonne National Laboratory. GeoSoilEnviroCARS is supported by the National Science Foundation - Earth Sciences (EAR - 1634415) and Department of Energy- GeoSciences (DE-FG0294ER14466). This research used resources of the Advanced Photon Source, a U.S. Department of Energy (DOE) Office of Science User Facility operated for the DOE Office of Science by Argonne National Laboratory under Contract No. DE-AC02-
06CH11357. Use of the GSECARS Raman Lab System was supported by the NSF MRI Proposal (EAR-1531583). This work made use of the IMSERC at Northwestern University, which has received support from the Soft and Hybrid Nanotechnology Experimental (SHyNE) Resource (NSF ECCS-1542205), the State of Illinois, and the International Institute for Nanotechnology (IIN). This work made use of the SPID facility of Northwestern University's NUANCE Center, which has received support from the Soft and Hybrid Nanotechnology Experimental (SHyNE) Resource (NSF ECCS-1542205); the MRSEC program (NSF DMR-1720139) at the Materials Research Center; the International Institute for Nanotechnology (IIN); the Keck Foundation; and the State of Illinois, through the IIN. This work made use of the Keck-II facility of Northwestern University's NUANCE Center, which has received support from the Soft and Hybrid Nanotechnology Experimental (SHyNE) Resource (NSF ECCS1542205); the MRSEC program (NSF DMR-1720139) at the Materials Research Center; the International Institute for Nanotechnology (IIN); the Keck Foundation; and the State of Illinois, through the IIN. M.C.W. is supported by the NSF Graduate Research Fellowship under grant DGE-1842165.

\section{REFERENCES}

(1) Li, H.; Wang, K.; Sun, Y.; Lollar, C. T.; Li, J.; Zhou, H.-C. Recent advances in gas storage and separation using metal-organic frameworks. Mater. Today 2018, 21, 108-121.

(2) Liao, Y.; Zhang, L.; Weston, M. H.; Morris, W.; Hupp, J. T.; Farha, O. K. Tuning ethylene gas adsorption via metal node modulation: $\mathrm{Cu}-\mathrm{MOF}-74$ for a high ethylene deliverable capacity. Chem. Commun. 2017, 53, 9376-9379.

(3) Kim, H.; Yang, S.; Rao, S. R.; Narayanan, S.; Kapustin, E. A.; Furukawa, H.; Umans, A. S.; Yaghi, O. M.; Wang, E. N. Water harvesting from air with metal-organic frameworks powered by natural sunlight. Science 2017, 356, 430-434.

(4) Denny, M. S.; Moreton, J. C.; Benz, L.; Cohen, S. M. Metal-organic frameworks for membrane-based separations. Nat. Rev. Mater. 2016, 1, 16078-16094.

(5) Trickett, C. A.; Osborn Popp, T. M.; Su, J.; Yan, C.; Weisberg, J.; Huq, A.; Urban, P.; Jiang, J.; Kalmutzki, M. J.; Liu, Q.; Baek, J.; HeadGordon, M. P.; Somorjai, G. A.; Reimer, J. A.; Yaghi, O. M. Identification of the strong Brønsted acid site in a metal-organic framework solid acid catalyst. Nat. Chem. 2019, 11, 170-176.

(6) Li, Z.; Peters, A. W.; Bernales, V.; Ortuño, M. A.; Schweitzer, N. M.; DeStefano, M. R.; Gallington, L. C.; Platero-Prats, A. E.; Chapman, K. W.; Cramer, C. J.; Gagliardi, L.; Hupp, J. T.; Farha, O. K. Metal-Organic Framework Supported Cobalt Catalysts for the Oxidative Dehydrogenation of Propane at Low Temperature. ACS Cent. Sci. 2017, 3, 31-38.

(7) Manna, K.; Ji, P.; Greene, F. X.; Lin, W. Metal-Organic Framework Nodes Support Single-Site Magnesium-Alkyl Catalysts for Hydroboration and Hydroamination Reactions. J. Am. Chem. Soc. 2016, 138, 7488-7491.

(8) Moghadam, P. Z.; Li, A.; Wiggin, S. B.; Tao, A.; Maloney, A. G. P.; Wood, P. A.; Ward, S. C.; Fairen-Jimenez, D. Development of a Cambridge Structural Database Subset: A Collection of Metal-Organic Frameworks for Past, Present, and Future. Chem. Mater. 2017, 29, 2618-2625.

(9) Howarth, A. J.; Liu, Y.; Li, P.; Li, Z.; Wang, T. C.; Hupp, J. T.; Farha, O. K. Chemical, thermal and mechanical stabilities of metalorganic frameworks. Nat. Rev. Mater. 2016, 1, 15018-15032.

(10) Bennett, T. D.; Simoncic, P.; Moggach, S. A.; Gozzo, F.; Macchi, P.; Keen, D. A.; Tan, J.-C.; Cheetham, A. K. Reversible pressureinduced amorphization of a zeolitic imidazolate framework (ZIF4). Chem. Commun. 2011, 47, 7983-7985.

(11) Chapman, K. W.; Halder, G. J.; Chupas, P. J. Pressure-Induced Amorphization and Porosity Modification in a Metal-Organic Framework. J. Am. Chem. Soc. 2009, 131, 17546-17547. 
(12) McKellar, S. C.; Sotelo, J.; Greenaway, A.; Mowat, J. P. S.; Kvam, O.; Morrison, C. A.; Wright, P. A.; Moggach, S. A. Pore Shape Modification of a Microporous Metal-Organic Framework Using High Pressure: Accessing a New Phase with Oversized Guest Molecules. Chem. Mater. 2016, 28, 466-473.

(13) Coudert, F.-X.; Fuchs, A. H. Computational characterization and prediction of metal-organic framework properties. Coord. Chem. Rev. 2016, 307, 211-236.

(14) Cavka, J. H.; Jakobsen, S.; Olsbye, U.; Guillou, N.; Lamberti, C.; Bordiga, S.; Lillerud, K. P. A New Zirconium Inorganic Building Brick Forming Metal Organic Frameworks with Exceptional Stability. J. Am. Chem. Soc. 2008, 130, 13850-13851.

(15) Valenzano, L.; Civalleri, B.; Chavan, S.; Bordiga, S.; Nilsen, M. H.; Jakobsen, S.; Lillerud, K. P.; Lamberti, C. Disclosing the Complex Structure of UiO-66 Metal Organic Framework: A Synergic Combination of Experiment and Theory. Chem. Mater. 2011, 23, 17001718.

(16) Peterson, G. W.; DeCoste, J. B.; Glover, T. G.; Huang, Y.; Jasuja, H.; Walton, K. S. Effects of pelletization pressure on the physical and chemical properties of the metal-organic frameworks $\mathrm{Cu}_{3}(\mathrm{BTC})_{2}$ and UiO-66. Microporous Mesoporous Mater. 2013, 179, 48-53.

(17) Wu, H.; Yildirim, T.; Zhou, W. Exceptional Mechanical Stability of Highly Porous Zirconium Metal-Organic Framework UiO66 and Its Important Implications. J. Phys. Chem. Lett. 2013, 4, 925930.

(18) Bennett, T. D.; Cheetham, A. K.; Fuchs, A. H.; Coudert, F.-X. Interplay between defects, disorder and flexibility in metal-organic frameworks. Nat. Chem. 2016, 9, 11-16.

(19) Dissegna, S.; Vervoorts, P.; Hobday, C. L.; Düren, T.; Daisenberger, D.; Smith, A. J.; Fischer, R. A.; Kieslich, G. Tuning the Mechanical Response of Metal-Organic Frameworks by Defect Engineering. J. Am. Chem. Soc. 2018, 140, 11581-11584.

(20) Rogge, S. M. J.; Wieme, J.; Vanduyfhuys, L.; Vandenbrande, S.; Maurin, G.; Verstraelen, T.; Waroquier, M.; Van Speybroeck, V. Thermodynamic Insight in the High-Pressure Behavior of UiO-66: Effect of Linker Defects and Linker Expansion. Chem. Mater. 2016 $28,5721-5732$.

(21) Schneider, C.; Bodesheim, D.; Ehrenreich, M. G.; Crocellà, V.; Mink, J.; Fischer, R. A.; Butler, K. T.; Kieslich, G. Tuning the Negative Thermal Expansion Behavior of the Metal-Organic Framework Cu3BTC2 by Retrofitting. J. Am. Chem. Soc. 2019, 141, 1050410509.

(22) Redfern, L. R.; Robison, L.; Wasson, M. C.; Goswami, S.; Lyu, J.; Islamoglu, T.; Chapman, K. W.; Farha, O. K. Porosity Dependence of Compression and Lattice Rigidity in Metal-Organic Framework Series. J. Am. Chem. Soc. 2019, 141, 4365-4371.

(23) Gao, H.; Wei, W.; Dong, L.; Feng, G.; Jiang, X.; Wu, R.; Lin, Z.; Li, W. Enhanced Framework Rigidity of a Zeolitic Metal-Azolate via Ligand Substitution. Crystals 2017, 7, 99-109.

(24) Hobday Claire, L.; Marshall Ross, J.; Murphie Colin, F.; Sotelo, J.; Richards, T.; Allan David, R.; Düren, T.; Coudert, F.-X.; Forgan Ross, S.; Morrison Carole, A.; Moggach Stephen, A.; Bennett Thomas, D. A Computational and Experimental Approach Linking Disorder, High-Pressure Behavior, and Mechanical Properties in UiO Frameworks. Angew. Chem., Int. Ed. 2016, 55, 2401-2405.

(25) Yot, P. G.; Yang, K.; Ragon, F.; Dmitriev, V.; Devic, T.; Horcajada, P.; Serre, C.; Maurin, G. Exploration of the mechanical behavior of metal organic frameworks UiO-66(Zr) and MIL-125(Ti) and their $\mathrm{NH}_{2}$ functionalized versions. Dalton Trans. 2016, 45, 4283 4288 .

(26) Tan, J. C.; Bennett, T. D.; Cheetham, A. K. Chemical structure, network topology, and porosity effects on the mechanical properties of Zeolitic Imidazolate Frameworks. Proc. Natl. Acad. Sci. U. S A. 2010, 107, 9938-9943.

(27) Moghadam, P. Z.; Rogge, S. M. J.; Li, A.; Chow, C.-M.; Wieme, J.; Moharrami, N.; Aragones-Anglada, M.; Conduit, G.; Gomez-Gualdron, D. A.; Van Speybroeck, V.; Fairen-Jimenez, D. Structure-
Mechanical Stability Relations of Metal-Organic Frameworks via Machine Learning. Matter 2019, 1, 219-234.

(28) Rogge, S. M. J.; Yot, P. G.; Jacobsen, J.; Muniz-Miranda, F.; Vandenbrande, S.; Gosch, J.; Ortiz, V.; Collings, I. E.; DevautourVinot, S.; Maurin, G.; Stock, N.; Van Speybroeck, V. Charting the Metal-Dependent High-Pressure Stability of Bimetallic UiO-66 Materials. ACS Mater. Lett. 2020, 2, 438-445.

(29) Bennett, T. D.; Tan, J.-C.; Moggach, S. A.; Galvelis, R.; MellotDraznieks, C.; Reisner, B. A.; Thirumurugan, A.; Allan, D. R.; Cheetham, A. K. Mechanical Properties of Dense Zeolitic Imidazolate Frameworks (ZIFs): A High-Pressure X-ray Diffraction, Nanoindentation and Computational Study of the Zinc Framework $\mathrm{Zn}(\mathrm{Im}) 2$, and its Lithium-Boron Analogue, LiB(Im)4. Chem. Eur. J. 2010, 16, 10684-10690.

(30) Yot, P. G.; Yang, K.; Guillerm, V.; Ragon, F.; Dmitriev, V.; Parisiades, P.; Elkaïm, E.; Devic, T.; Horcajada, P.; Serre, C.; Stock, N.; Mowat, J. P. S.; Wright, P. A.; Férey, G.; Maurin, G. Impact of the Metal Centre and Functionalization on the Mechanical Behaviour of MIL-53 Metal-Organic Frameworks. Eur. J. Inorg. Chem. 2016, 2016, 4424-4429.

(31) Ayoub, G.; Islamoglu, T.; Goswami, S.; Friščić, T.; Farha, O. K. Torsion Angle Effect on the Activation of UiO Metal-Organic Frameworks. ACS Appl. Mater. Interfaces 2019, 11, 15788-15794.

(32) Islamoglu, T.; Ray, D.; Li, P.; Majewski, M. B.; Akpinar, I.; Zhang, X.; Cramer, C. J.; Gagliardi, L.; Farha, O. K. From Transition Metals to Lanthanides to Actinides: Metal-Mediated Tuning of Electronic Properties of Isostructural Metal-Organic Frameworks. Inorg. Chem. 2018, 57, 13246-13251.

(33) Lammert, M.; Wharmby, M. T.; Smolders, S.; Bueken, B.; Lieb, A.; Lomachenko, K. A.; Vos, D. D.; Stock, N. Cerium-based metal organic frameworks with UiO-66 architecture: synthesis, properties and redox catalytic activity. Chem. Commun. 2015, 51, 1257812581.

(34) Holtgrewe, N.; Greenberg, E.; Prescher, C.; Prakapenka, V. B.; Goncharov, A. F. Advanced integrated optical spectroscopy system for diamond anvil cell studies at GSECARS. High Press. Res. 2019, 39, 457-470.

(35) Dovesi, R.; Orlando, R.; Civalleri, B.; Roetti, C.; Saunders, V.; Zicovich-Wilson, C. CRYSTAL: A computational tool for the ab initio study of the electronic properties of crystals. Z. Kristallogr. - Cryst. Mater. 2005, 220, 571-573.

(36) Gatti, C.; Saunders, V. R.; Roetti, C. Crystal field effects on the topological properties of the electron density in molecular crystals: The case of urea. J. Chem. Phys. 1994, 101, 10686-10696.

(37) Muñoz Ramo, D.; Gavartin, J. L.; Shluger, A. L.; Bersuker, G. Spectroscopic properties of oxygen vacancies in monoclinic $\mathrm{HfO}_{2}$ calculated with periodic and embedded cluster density functional theory. Phys. Rev. B 2007, 75, 205336.

(38) Graciani, J.; Márquez, A. M.; Plata, J. J.; Ortega, Y.; Hernández, N. C.; Meyer, A.; Zicovich-Wilson, C. M.; Sanz, J. F. Comparative Study on the Performance of Hybrid DFT Functionals in Highly Correlated Oxides: The Case of $\mathrm{CeO}_{2}$ and $\mathrm{Ce}_{2} \mathrm{O}_{3}$. J. Chem. Theory Comput. 2011, 7, 56-65.

(39) Perdew, J. P.; Ruzsinszky, A.; Csonka, G. I.; Vydrov, O. A.; Scuseria, G. E.; Constantin, L. A.; Zhou, X.; Burke, K. Restoring the Density-Gradient Expansion for Exchange in Solids and Surfaces. Phys. Rev. Lett. 2008, 100, 136406-136409.

(40) Grimme, S. Semiempirical GGA-type density functional constructed with a long-range dispersion correction. J. Comput. Chem. 2006, 27, 1787-1799.

(41) Monkhorst, H. J.; Pack, J. D. Special points for Brillouin-zone integrations. Phys. Rev. B 1976, 13, 5188-5192.

(42) Shearer, G. C.; Chavan, S.; Ethiraj, J.; Vitillo, J. G.; Svelle, S.; Olsbye, U.; Lamberti, C.; Bordiga, S.; Lillerud, K. P. Tuned to Perfection: Ironing Out the Defects in Metal-Organic Framework UiO-66. Chem. Mater. 2014, 26, 4068-4071.

(43) Zhou, M.; Wang, K.; Men, Z.; Sun, C.; Li, Z.; Liu, B.; Zou, G.; Zou, B. Pressure-induced isostructural phase transition of a metal- 
organic framework $\mathrm{Co}_{2}\left(4,4^{\prime}-\mathrm{bpy}\right)_{3}\left(\mathrm{NO}_{3}\right)_{4} \cdot \mathrm{xH}_{2} \mathrm{O}$. CrystEngComm 2014, 16, 4084-4087.

(44) Su, Z.; Miao, Y.-R.; Zhang, G.; Miller, J. T.; Suslick, K. S. Bond breakage under pressure in a metal organic framework. Chem. Sci. 2017, 8, 8004-8011.

(45) Taddei, M.; Tiana, D.; Casati, N.; van Bokhoven, J. A.; Smit, B.; Ranocchiari, M. Mixed-linker UiO-66: structure-property relationships revealed by a combination of high-resolution powder X-ray diffraction and density functional theory calculations. Phys. Chem. Chem. Phys. 2017, 19, 1551-1559.

(46) Marrocchelli, D.; Bishop, S. R.; Tuller, H. L.; Yildiz, B. Understanding Chemical Expansion in Non-Stoichiometric Oxides: Ceria and Zirconia Case Studies. Adv. Funct. Mater. 2012, 22, 1958-1965.
(47) Muhich, C. L. Re-Evaluating $\mathrm{CeO}_{2}$ Expansion Upon Reduction: Noncounterpoised Forces, Not Ionic Radius Effects, Are the Cause. J. Phys. Chem. C 2017, 121, 8052-8059.

(48) Syzgantseva, M. A.; Ireland, C. P.; Ebrahim, F. M.; Smit, B.; Syzgantseva, O. A. Metal Substitution as the Method of Modifying Electronic Structure of Metal-Organic Frameworks. J. Am. Chem. Soc. 2019, 141, 6271-6278.

(49) Islamoglu, T.; Atilgan, A.; Moon, S.-Y.; Peterson, G. W.; DeCoste, J. B.; Hall, M.; Hupp, J. T.; Farha, O. K. Cerium(IV) vs Zirconium(IV) Based Metal-Organic Frameworks for Detoxification of a Nerve Agent. Chem. Mater. 2017, 29, 2672-2675.

(50) Rieth, A. J.; Wright, A. M.; Dincă, M. Kinetic stability of metal-organic frameworks for corrosive and coordinating gas capture. Nat. Rev. Mater. 2019, 4, 708-725.

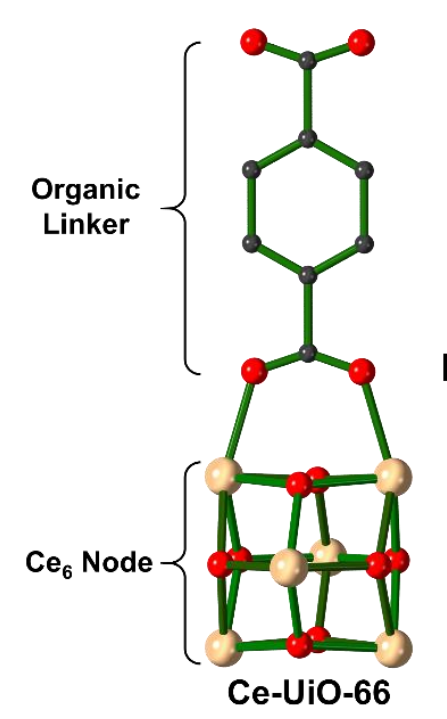

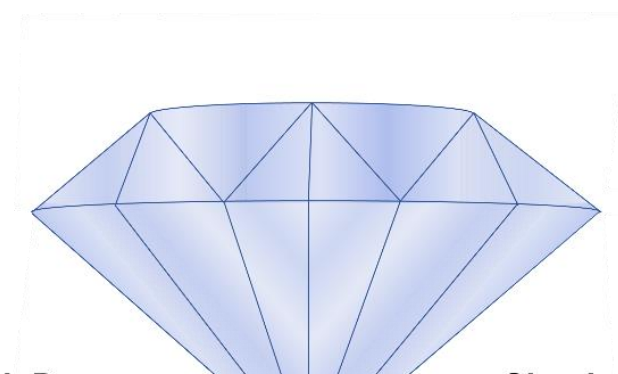

High Pressure

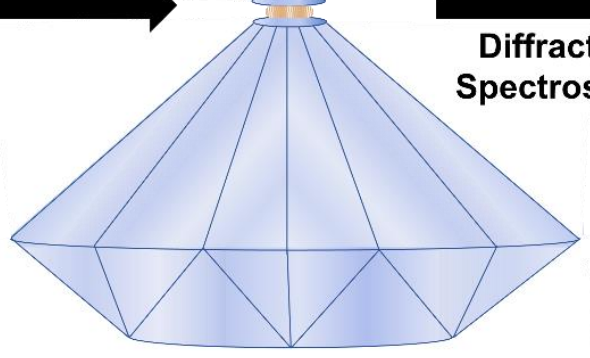

Simulation

Diffraction, pectroscopy

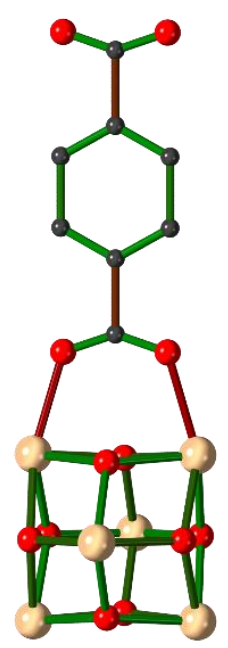

Most Compressible

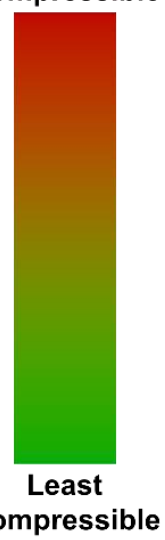

